\title{
Gleichstellung nur nach Schaffung rechtlicher Rahmenbedingungen
}

\section{Dr. Volkmar Schöneburg}

Justizminister des Landes Brandenburg, Potsdam

Meine Damen und Herren,

ich begrüße Sie im Namen der Landesregierung und auch in meinem Namen recht herzlich hier in Potsdam. Es ist wunderbar, dass Ihr 39. Kongress heute in meiner Heimatstadt stattfindet. Dieses Grußwort ist für mich daher ein Heimspiel. Hier in dieser Halle habe ich, bevor ich Minister wurde, Lesungen mitorganisiert, unter anderem mit Daniela Dahn, die sich auch in der DDR und in der Wendezeit für Frauenrechte eingesetzt hat. Hier habe ich mit ihr ein Interview zu einem ihrer Bücher geführt. Aber von so viel Frauenpower heute bin ich doch etwas eingeschüchtert. Wenn Juristinnen eine Tagung abhalten, scheint es mir anmaßend, wenn ich hier als Minister versuche, kluge Sätze zu präsentieren. Deswegen werde ich mich relativ kurz fassen, Sie wirklich nur begrüßen und nur ganz wenige Gedanken äußern.

Eines der wichtigsten Bücher in meiner wissenschaftlichen Karriere vor 1989/90 war ein Buch, das im Nomos Verlag erschienen ist. Um in der DDR an Bücher heranzukommen, die im Westen Deutschlands herausgegeben worden sind, musste man diese Bücher rezensieren. Dann erhielt man sie und konnte die eigene Bibliothek auffrischen. Eines dieser Bücher, die ich rezensiert habe, war 1988 das Buch „Streitbare Juristen. Eine andere Tradition“. Ich habe es mir gestern noch einmal zur Hand genommen und dachte, ich schaue nach, wie viele streitbare Juristinnen denn in diesem Band, der ja 41 Juristenporträts des 18., 19. und 20. Jahrhunderts enthält, die - so schrieben es die Herausgeber in der Einleitung - auf Seiten derjenigen standen, denen das Recht oft zum Unrecht ausschlug. Dazu gehörten Persönlichkeiten wie Gustav Radbruch, der Anfang der Zwanzigerjahre Wesentliches in die Wege leitete und den Zugang der Frauen zu den Berufen der Rechtspflege gesetzlich fixierte oder das jedenfalls als Reichsjustizminister auf den Weg brachte. Es waren auch Karl Liebknecht, Wolfgang Abendroth oder Fritz Bauer enthalten. Aber es wurden sage und schreibe nur drei Frauen in dem Band porträtiert: Anita Augspurg, eine der ersten feministischen Juristinnen, die 1897 in Zürich promovierte und sich ganz stark an der Diskussion zum Entstehen des Bürgerlichen Gesetzbuches beteiligte, um dort Benachteiligungen von Frauen auszuräumen. Die zweite war Helene Stöcker, eine Frauenrechtlerin, die eigentlich promovierte Literaturwissenschaftlerin war. Sie setzte sich in der Frauenrechtsbewegung insbesondere für eine Reformierung oder Abschaffung des $\mathbb{2}$ 218, 219 StGB ein sowie für eine Abschaffung des $\mathbb{1} 175 \mathrm{StGB}$ und wandte sich auch gegen dessen Ausweitung auf Frauen. Die dritte war Elisabeth Selbert, im Parlamentarischen Rat eine von vier Frauen. Sie war maßgeblich für die Aufnahme der Formulierung
„Frauen und Männer sind gleichberechtigt“ in das Grundgesetz verantwortlich.

Nur drei Frauen wurden in diesem Buch dargestellt: d.h. selbst in einer sich emanzipatorisch gebenden Bewegung, in der linken Bewegung, gelang es Frauen in den juristischen Berufen oder mit juristischen Themen nur allmählich und langsam sich durchzusetzen. Sie erkämpften sich den entsprechenden Raum auch dort erst, nachdem die rechtlichen Rahmenbedingungen geschaffen worden waren. Es war ein langer konfliktreicher Prozess, bis Frauen entsprechende Positionen erlangten. Insofern finde ich, dass dieses Thema „Gleichberechtigung im Öffentlichen Dienst", das sie hier bei Ihrem 39. Bundeskongress bearbeiten, völlig legitim ist - auch wenn diese Aussage jetzt doch ein bisschen altväterlich wirkt. Ich unterstütze Sie sehr dabei, erstens zu evaluieren, was die rechtlichen Regelungen gebracht haben und zum Teil eben auch dafür zu plädieren, dass Quoten eingerichtet oder Strukturen geschaffen werden, die wirklich der Gleichberechtigung auf allen Gebieten zum Durchbruch verhelfen. Und ich weiß, wovon ich rede, denn ich komme heute gerade aus Magdeburg von der Sonder-Justizministerkonferenz. Auch unter den Justizministern und Justizministerinnen sind die Frauen, wenngleich sie dort sehr aktiv auftreten und eine starke Stimme haben, immer noch in der Minderheit. Also selbst in den Spitzen haben wir mehr eine Männer- als eine Frauengesellschaft und das ist, um mit Klaus Wowereit zu sprechen „Nicht gut so".

Wir haben auch während der letzten ordentlichen Justizministerkonferenz im Mai 2011 in Halle (Saale) über Frauen in Vorständen von Betrieben, von privatwirtschaftlichen Unter-

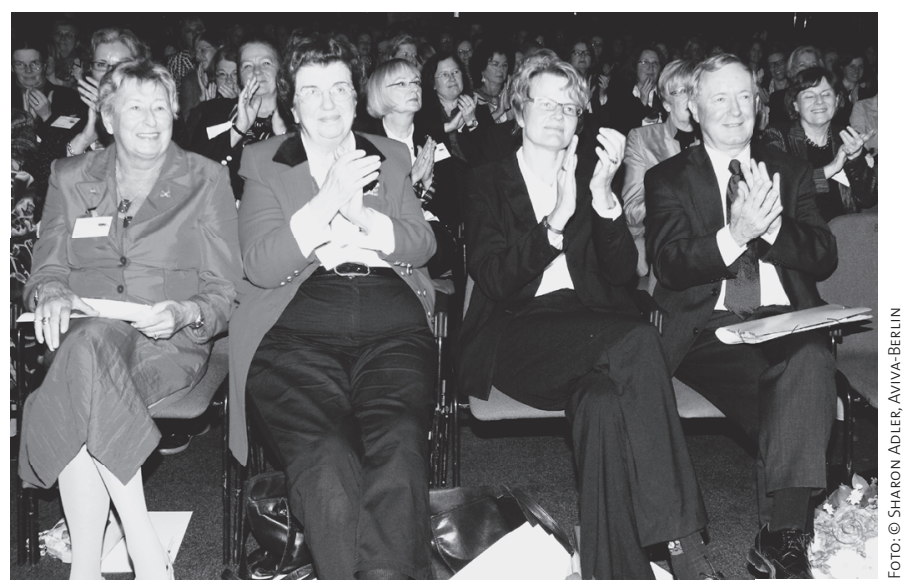

A Bei der Eröffnung des Kongresses am 22. September 2011 in der von KarlFriedrich Schinkel entworfenen WaschhausARENA im Areal Schiffbauergasse, Potsdam in der ersten Reihe (v.l.n.r.): Renate Damm und Dr. Lore Maria Peschel-Gutzeit (Ehrenpräsidentinnen des djb), Prof. Dr. Susanne Baer, LL.M. (Richterin des Bundesverfassungsgerichts, Festrednerin) und Dr. Max Stadler, MdB (Parl. Staatssekretär im Bundesministerium der Justiz). 


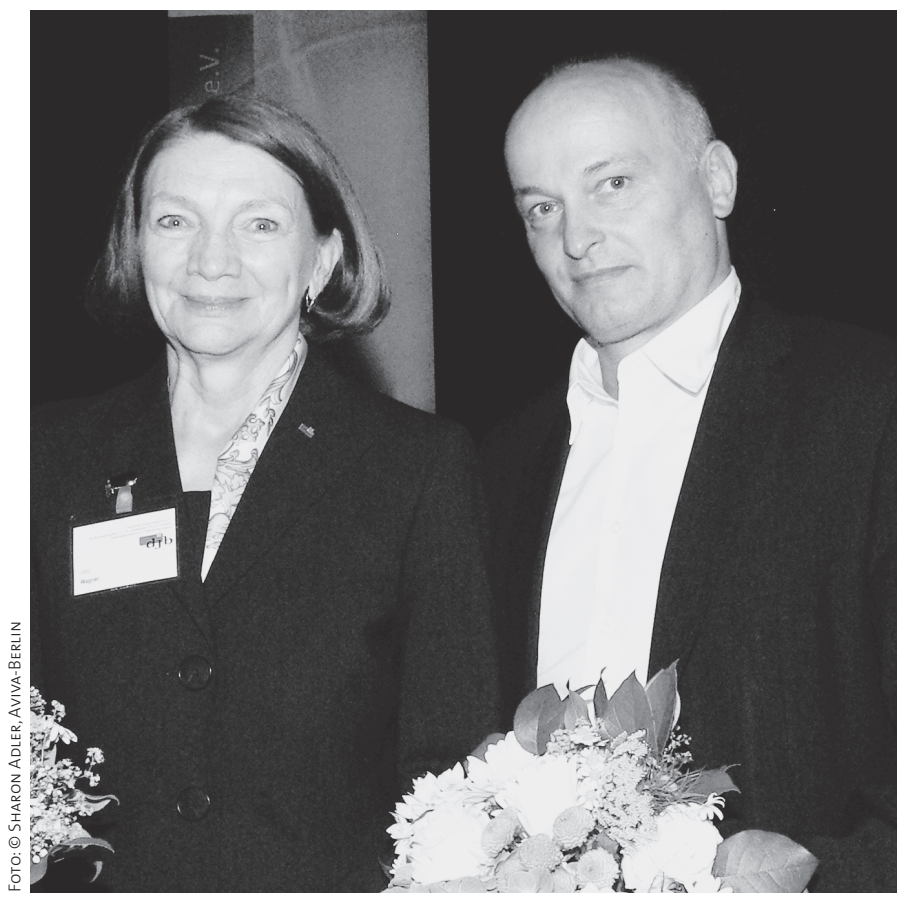

A Jutta Wagner überreicht Dr. Volkmar Schöneburg (Justizminister des Landes Brandenburg) als Dank für ein Grußwort an die Teilnehmerinnen und Teilnehmer des 39. Bundeskongresses einen Blumenstrauß.

nehmungen diskutiert. Es gab immer die zwei konträren Positionen: die einen, die sagten, man müsse die Unternehmen dazu zwingen, eine Quote durchzusetzen. Das ist auch meine Position. Außerdem hört man die mehr von freien Demokraten geäußerte Auffassung, dass man die Frauen in Führungs- positionen einem gewissen Selbstlauf überlassen müsse. Nein, ich denke, man muss dazu strukturelle Entscheidungen treffen, diese entsprechend fixieren und dann durchsetzen. Es ist schwer genug, wie die streitbaren Juristinnen gezeigt haben, die entsprechende Quote dann auch tatsächlich zu verwirklichen.

Eine zweite Anmerkung sei mir noch kurz gestattet. Ich freue mich sehr, dass es dem djb gelungen ist, die Ausstellung zu den Juristinnen aus der DDR auf die Beine zu stellen, die morgen Abend im Landgericht Potsdam eröffnet wird. Ich finde, dass ein differenzierter Blick auf Juristinnen in der DDR in zweierlei Hinsicht eine wichtige Sache ist: Man kann anhand der Ausstellung nachvollziehen, dass die Justiz in der DDR aus den unterschiedlichsten bekannten Gründen nicht so eine Rolle gespielt hat wie im Westen Deutschlands, dass sie eine Unterdrückungsfunktion hatte und die Strafjustiz hart war, dass zum Teil auch Unrecht in Rechtsform produziert wurde. Dafür steht das Porträt von Hilde Benjamin, wobei man auch sie nicht allein darauf reduzieren kann. Andererseits gelang der DDR eine bedeutend höhere Frauenquote unter den Juristen als parallel dazu der Bundesrepublik. Ich habe gestern Abend noch Uwe Wesels „Recht, Unrecht und Gerechtigkeit. Von der Weimarer Republik bis heute“ gelesen, der sagte, das habe auch mit dazu geführt, dass die Justiz im Westen etwas männlicher und härter und im Osten etwas weiblicher und außerhalb der politischen Konflikte auf mehr Harmonie ausgerichtet gewesen sei. Letzteres habe der Justiz gut zu Gesicht gestanden. Vielleicht kann man aus der Ausstellung auch dazu etwas lernen. Ich wünsche Ihnen eine wunderbare Tagung! Danke.

\section{Gleichberechtigung ist ein Menschenrecht}

\section{Dr. Max Stadler, MdB}

Parlamentarischer Staatssekretär bei der Bundesministerin der Justiz, Berlin

\section{Meine Damen und Herren,}

der Weg von Berlin aus dem Reichstag hierher war kurz, der Weg von dem sitzenden Publikum auf die Bühne ist relativ lang. Gleichwohl habe ich auch diesen Weg noch pünktlich geschafft und ich bedanke mich sehr herzlich für die Einladung, sehr geehrte Frau Präsidentin Wagner, sehr geehrter Herr Minister, Herr Oberbürgermeister, meine sehr geehrten Damen und Herren.

Eva Spagna, die Sängerin, die einen so schönen kulturellen Rahmen für die Veranstaltung bietet, meinte vorhin, beim Papst seien wahrscheinlich viele Männer gewesen. Das ist natürlich richtig, aber nur die halbe Wahrheit. Es waren auch viele Frauen dort. Manche haben sogar begeistert applaudiert. So zum Beispiel Claudia Roth und Renate Künast, weil der Papst zur allgemeinen Überraschung die ökologische Bewegung sehr gelobt hat. Ebenfalls war überraschend, dass er sich in seiner Rede vorhin im Reichstag mit rechtsphilosophischen Fragen befasste. Er hat sich auseinandergesetzt mit Hans Kelsen, der bekanntlich der Begründer der reinen Rechtslehre ist, der Schöpfer der österreichischen Verfassungsgerichtsbarkeit und ein bedeutender Rechtspositivist. Während der Papst sich als Verfechter des Naturrechts dargestellt hat. Ich glaube, egal welchen Begründungsstrang man verfolgt, eines steht fest: Gleichberechtigung ist ein unverbrüchliches Menschenrecht. Das werden Rechtspositivisten und Vertreter des Naturrechts gleichermaßen so sehen. Ich will mich nun in diese Fragen nicht weiter einmischen, denn wir hören jetzt gleich einen Vortrag von Frau Prof. Dr. Baer, die womöglich auch auf diese Themen eingeht, so dass ich es in meinem Grußwort dabei belassen will, einige wenige Fakten zu nennen, die zu Ihrem Thema passen. Deswegen, Frau Präsidentin, antworte ich jetzt auch nicht auf die rechtspolitischen Probleme, die Sie angerissen haben. Dafür ist ein Grußwort wohl nicht der richtige Ort.

Aber Ihr Kongress hat mir Anlass gegeben, im eigenen Haus und im eigenen Geschäftsbereich nachzuforschen, ob wir uns denn einigermaßen als Vorreiter für Gleichberechti- 